\title{
Medir desempeño del servicio de bancos detallistas en México: una adaptación del Servperf
}

\author{
Measuring service performance of retail banks in Mexico: \\ A Servperf adaptation
}

\author{
Jorge Vera* y Andrea Trujillo \\ Tecnológico de Monterrey, México
}

Recibido el 13 de mayo del 2016; aceptado el 27 de octubre del 2016

Disponible en Internet el 27 de septiembre de 2018

\section{Resumen}

El propósito es ofrecer un instrumento para medir desempeño del servicio de bancos detallistas en México. Este instrumento está basado en la escala SERVPERF propuesta por Cronin y Taylor (1992). El artículo se enmarca dentro del área de medición de la calidad y del desempeño del servicio. Se parte de una adaptación previa en inglés del SERVPERF a esta industria. Se narra el proceso de adaptación de los reactivos al idioma español en el contexto mexicano. Para esta adaptación se utilizó tanto consulta con especialistas, como entrevistas a profundidad con clientes de bancos, para verificar que entendieran la redacción de los reactivos. Se presenta una prueba empírica del cuestionario con $n=201$ clientes de bancos detallistas. Se realizaron diversas pruebas para corroborar confiabilidad y validez del instrumento. Se muestra que la escala cumple con criterios de validez convergente y discriminante mediante análisis factorial y análisis estructural confirmatorio. Se analizan y se discuten las dimensiones de la escala donde estas se comparan con planteamientos anteriores. Adicionalmente se lleva a cabo una prueba de validez predictiva mediante análisis de regresión.

Códigos JEL: M30, M31, M39.

Palabras clave: Desempeño del servicio; Calidad del servicio; Servicio bancario detallista; Banco detallista.

\footnotetext{
*Autor para correspondencia.

Correo electrónico: jorge.vera@itesm.mx (J. Vera)

La revisión por pares es responsabilidad de la Universidad Nacional Autónoma de México.
} 


\begin{abstract}
The purpose of this work is to offer a scale to measure service performance of retail banks in Mexico. It is based on the SERVPERF scale by Cronin and Taylor (1992). The article is framed in the service quality and performance literature. This instrument is initially based on a previous adaptation of the SERVPERF scale in English to retail banks. It is explained how the items were contextually adapted to the Spanish language. For a correct translation-adaptation interviews with specialists and clients were carried over. This procedure helped to ensure that the writing of the items was understandable end to verify that they were measuring what was intended. The result of an $n=201$ retail bank clients empirical test is presented. Several test to verify reliability and validity were performed. Using an factorial analysis and a confirmatory structural analysis it is presented how the scale accomplish convergent and discriminant validity criteria as well. In comparison with previous approaches, the dimensionality of the scale is analyzed. Also, an assessment of the scale's predictive validity was performed.
\end{abstract}

JEL Classification: M30, M31, M39.

Keywords: Accruals: Service performance; Service quality; Retail bank service; Retail bank.

\title{
Introducción
}

La calidad en el servicio, entendida como "un juicio global o actitud relacionada con la superioridad del servicio" (Parasuraman et al., 1988, p.16), es un tema que interesa tanto a académicos, por ser un constructo multidimensional de alto valor en investigación de comportamiento del consumidor (Grönroos, 1984; Parasuraman et al., 1985), como a los tomadores de decisiones de las empresas, esto por el impacto que tiene en la retención de clientes (Zeitham, 2000).

La calidad en el servicio se ha venido estudiando desde hace más de tres décadas, sin embargo, la medición de la misma sigue siendo un tema que mantiene ocupados a los investigadores (Simmers y Keith, 2015; Rauch, et al., 2015; Cater-steel y Lepmets, 2014; Keith y Simmers, 2013; Jain et al., 2013). A finales de los 80's se consideraba al SERVQUAL como un instrumento único para medir calidad en el servicio en cualquier tipo de empresa y en cualquier país. Sin embargo, las críticas al mismo sugieren que es preferible tener una escala particular para cada tipo de servicio (Ladhari, 2008), ya que las características de cada uno son diferentes y eso hace que se agreguen, se recorten o se rediseñen reactivos para cada sector. En los años recientes se han diseñado escalas específicas para restaurantes (Andaleeb y Conway, 2006; Antun, et al., 2010), hospitales (Padma et al., 2015), hoteles (Akbaba, 2006), entre otros. También se han diseñado escalas para culturas específicas como la paquistaní (Raajpoot, 2004) y la árabe (Jabnoun, y Khalifa, 2005). De ahí la necesidad de adaptar escalas para cada industria en un contexto específico. De esta manera, El propósito principal de este artículo es ofrecer una escala que mida la calidad del servicio diseñada específicamente para el sector bancario detallista en sucursal en el contexto mexicano.

Se propone una escala para este tipo de servicio bancario dada la relevancia económica para los clientes (ya que trabaja directamente con su dinero). Así, la elección y permanencia en un banco se debe en gran parte a la evaluación que hace el usuario sobre la institución donde maneja su dinero; en diversos estudios se ha comprobado la relación que existe entre la calidad en el servicio y la permanencia en un banco (Belás y Gabcová, 2016; Mittal et al., 
2015; Kaur, y Kiran, 2015). La escala es útil para la banca en México ya que, de acuerdo con la Comisión Nacional Bancaria y de Valores (2016) se reportó la existencia de 12,234 sucursales bancarias en diciembre del 2015. Así, según los datos de la Encuesta Nacional de Inclusión Financiera (INEGI, 2012), el 97\% de la población adulta tiene acceso a algunos servicios bancarios, ya sea a través de las sucursales, cajeros automáticos, corresponsalías o puntos de venta.

La escala presentada en este artículo parte de una escala diseñada en inglés para medir el desempeño de bancos (Ladhari, et al., 2011), dicha escala es una adaptación del SERVPERF al contexto bancario. Y lo que se propone es un ajuste a dicha escala para que cada reactivo conserve su sentido original pero entendido en la realidad mexicana. Este estudio contribuye al entendimiento de las dimensiones que constituyen la calidad en el servicio en los bancos, además de que permite a los gerentes tener un instrumento con el que puedan evaluar aspectos específicos sobre los cuáles tomar decisiones.

En las siguientes secciones de este artículo se hace una revisión de los instrumentos de los que deriva la presente escala, para posteriormente presentar los análisis por los que pasó la misma para comprobar su validez y confiabilidad, finalmente se discuten los hallazgos y se plantean las dimensiones y reactivos finales de la misma.

\section{Calidad del servicio versus desempeño del servicio}

La calidad del servicio ha sido entendida como un constructo multidimensional. Parasuramen et al. (1985) proponen el SERVQUAL, en esta primera escala generalizable de calidad del servicio, se identificaron cinco dimensiones: tangibles, fiabilidad, capacidad de respuesta, seguridad y empatía. Los tangibles son los aspectos físicos que el cliente percibe en la organización. La fiabilidad se refiere a la habilidad de desarrollar el servicio prometido precisamente como se pactó y con exactitud. La capacidad de respuesta es la disposición inmediata para atender a los clientes y dar un pronto servicio La seguridad hace referencia al conocimiento y cortesía de los empleados y su habilidad para comunicarse e inspirar confianza, de manera que el cliente está seguro de recibir siempre lo mismo en una empresa. Y la empatía se refiere a proveer cuidados y atención individualizada a los clientes

El diseño del SERVQUAL estuvo basado en la premisa de que la calidad en el servicio puede ser vista como el grado y la dirección de discrepancia entre las expectativas y la percepción de lo que en realidad recibe el cliente. De ahí que el SERVQUAL se compone de dos partes una donde se pregunta sobre las expectativas -entendiéndolas como las predicciones que hace el cliente sobre el servicio- y otra sobre la percepción del resultado obtenido -es decir del desempeño de la empresa-. Sin embargo, una década después del nacimiento del SERVQUAL, Cronin y Taylor (1992 y 1994) hicieron diversos estudios para probar que no era necesario tener ítems que midieran las expectativas, puesto que, de manera natural, el cliente siempre evalúa a partir de una predicción que él mismo hace. Sus hallazgos demostraron que se puede medir la calidad en el servicio utilizando sólo la segunda parte propuesta en el SERVQUAL, es decir, midiendo la percepción que el cliente tiene del desempeño de la empresa, este nuevo instrumento fue llamado SERVPERF (pues toma su nombre del término service performance). Jain y Gupta (2004) señalan que, metodológicamente, el SERVPERF representa una mejora sobre el SERVQUAL, no sólo por ser un instrumento más parsimonioso por la considerable reducción en el número de reactivos, sino también por explicar una mayor varianza en la medición de la calidad general del servicio. Su validez ha sido comprobada también por otros 
autores (Brady et al., 2002; Carrillat et al., 2007; Radomir et al., 2012), por lo que en este estudio se realiza la medición siguiendo la propuesta de Cronin y Taylor (1994). La medición del desempeño del servicio, basándose en el enfoque de medición exclusiva del desempeño de los atributos propuesto por Cronin y Taylor (1992), también ha dado resultados favorables en pruebas de confiabilidad y validez en el sector de bancos detallistas (Karatepe et al., 2005; Culiberg y Rojsek, 2010). Siendo el enfoque SERVQUAL/SERVPERF uno que fue propuesto originalmente conformado por cinco dimensiones (ya mencionadas), en el desarrollo de instrumentos de medición de este tipo para diversas industrias se han propuesto dimensiones adicionales donde se miden aspectos específicos de cada tipo de industria. De esta manera, Culiberg y Rojsek (2010) probaron empíricamente, con éxito, la inclusión de una dimensión adicional que llamaron "accesibilidad al banco" (que tan fácil es llegar a él por ubicación y horario). Esta dimensión no fue considerada en este estudio, ya que el propósito era adaptar los atributos originales, sin embargo, al final de este artículo se hacen recomendaciones al respecto y se sugieren reactivos.

\section{Adaptación de la escala al idioma español}

Para la generación de esta escala se partió de una versión previa en idioma inglés (una adaptación del SERVPERF a bancos de servicio detallista). Así, se llevó a cabo un proceso de traducción y adaptación al idioma español. Este proceso involucró tres tipos de actividades: primero, una cuidadosa adaptación inicial al español del sentido original de los reactivos en inglés; luego, consultas con académicos mexicanos versados en el manejo del idioma inglés que propusieran correcciones; posteriormente, consultas con especialistas en mercadotecnia para ayudar a asegurar la validez de contenido. Estas tres actividades se detallan a continuación.

Para la traducción inicial de los veintidós reactivos se trató de evitar el uso de traducciones literales palabra por palabra. Como lo que se buscaba era emular el sentido original del significado de los reactivos, las traducciones consistieron en interpretaciones conceptuales de lo que cada reactivo implicaba para posteriormente elaborar en español ese mimo contenido conceptual. Esto ayudó a evitar ambigüedades e imprecisiones por el uso de términos fuera de contexto. La versión preliminar de los reactivos fue sometida al escrutinio de dos académicos mexicanos que contaban con un buen dominio del idioma inglés (con puntaje TOEFL superior a 600 puntos). En este escrutinio, a dichos jueces se les pidió comparar la versión original de la escala en inglés con la adaptación inicial al español. En este proceso debían identificar posibles errores de adaptación de un idioma a otro. Con sus comentarios, se realizaron ajustes a la redacción en español de los reactivos. Finalmente, para ayudar a la validez de contenido de los reactivos se solicitó a dos académicos con posgrados en mercadotecnia, profesores de esta área y familiarizados, tanto con estudios sobre comportamiento del consumidor, como con estudios sobre calidad en el servicio, que revisaran los reactivos haciendo un juicio sobre la variable que estaría midiendo cada uno de estos. Con base en este juicio se les solicitó identificar posibles puntos de mejora en la redacción de los reactivos. Con esta información nuevamente se realizaron ajustes a las redacciones de los ítems. 
Tabla 1

Declaraciones de los reactivos finales obtenidos del proceso de adaptación al español

$\begin{array}{ll}\text { sqb1 } & \text { El banco XYZ tiene un equipamiento que se ve moderno. } \\ \text { sqb2 } & \text { Las instalaciones del banco XYZ son visualmente atractivas. } \\ \text { sqb3 } & \text { Los empleados del banco XYZ presentan una apariencia muy bien cuidada. } \\ \text { sqb4 } & \text { La folletería y otros materiales impresos del banco XYZ son visualmente atractivos. } \\ \text { sqb5 } & \text { Cuando el banco XYZ promete hacer algo en cierto tiempo, lo cumple. } \\ \text { sqb6 } & \text { Cuando usted tiene un problema, el banco XYZ muestra un interés sincero en resolverlo. } \\ \text { sqb7 } & \text { El banco XYZ realiza bien el servicio a la primera vez. } \\ \text { sqb8 } & \text { El banco XYZ cumple con sus servicios en el tiempo prometido. } \\ \text { sqb9 } & \text { El banco XYZ busca siempre en no cometer errores. } \\ \text { sqb10 } & \text { Los empleados del banco XYZ le dicen exactamente en qué tiempo se cumplirá el servicio. } \\ \text { sqb11 } & \text { Los empleados del banco XYZ le brindan un servicio rápido. } \\ \text { sqb12 } & \text { Los empleados del banco XYZ siempre están dispuestos a ayudarlo. } \\ \text { sqb13 } & \text { Los empleados del banco XYZ siempre tienen tiempo de responder a sus solicitudes. } \\ \text { sqb14 } & \text { El comportamiento de los empleados del banco XYZ genera confianza en los clientes. } \\ \text { sqb15 } & \text { Se siente seguro al realizar transacciones en el banco XYZ. } \\ \text { sqb16 } & \text { Los empleados del banco XYZ son siempre corteses con usted. } \\ \text { sqb17 } & \text { Los empleados del banco XYZ tienen el conocimiento necesario para responder a sus preguntas. } \\ \text { sqb18 } & \text { El banco XYZ le brinda atención personalizada. } \\ \text { sqb19 } & \text { El banco XYZ tiene horarios de atención convenientes para sus clientes. } \\ \text { sqb20 } & \text { El banco XYZ tiene empleados que lo atienden personalmente. } \\ \text { sqb21 } & \text { Al banco XYZ realmente le importan sus necesidades. } \\ \text { sqb22 } & \text { Los empleados del banco XYZ entienden sus necesidades específicas. }\end{array}$

Fuente: elaboración propia

Con esta versión de la escala se implementaron dos pruebas piloto con clientes de bancos detallistas. Cada prueba piloto incluyó diez clientes de este tipo de bancos. En estas pruebas se pidió a los respondientes que contestaran los reactivos evaluando al banco que utilizan principalmente. Después que hubieron terminado de responder a los reactivos, se les entrevistó a profundidad sobre qué términos o aspectos de las redacciones de los reactivos no entendieron y pidiéndoles sugerencias sobre cómo podían quedar escritos de forma más clara. Primero se llevó a cabo un grupo de entrevistas con base en las cuales se hicieron, nuevamente, correcciones a los reactivos. Posteriormente, se realizaron un segundo grupo de entrevistas procediendo de la misma forma.

Con todo este proceso se obtuvo la versión final del cuestionario que fue sometida a una prueba empírica para determinar si esta escala cumplía tanto con criterios de confiabilidad como de validez. En la Tabla 1 se muestran las declaraciones de los reactivos obtenidos del proceso de adaptación al español. En el Apéndice 1 se puede apreciar el cuestionario en el formato con el que fue puesto a prueba. En el instrumento estas declaraciones van asociadas a escalamientos de actitud que van de 1 a 7 , donde 1 se vincula a "totalmente en desacuerdo" y 7 a "totalmente de acuerdo".

\section{Metodología de la prueba empírica}

Se les pidió a $n=250$ clientes de bancos detallistas que respondieran la escala. Estos clientes fueron seleccionados mediante un muestreo por conveniencia. Esta selección de respondientes se hizo mediante las redes de conocidos del equipo de entrevistadores que nos apoyaron (ver agradecimientos). No obstante, se siguió un proceso riguroso de selección que ayudara a validar la muestra y evitar sesgos. De esta forma los participantes debían cumplir con los criterios que se detallan a continuación: 
Mayores de edad que cuenten con algún servicio bancario en México (cuenta de ahorros, cuenta de cheques y/o tarjeta de crédito). Personas distribuidas homogéneamente entre 20 y 60 años de edad evitando tener sobrerrepresentación por grupos específicos de edad. Se controló la distribución por género tratando de obtener mitad de respondientes mujeres y la otra mitad hombres. De forma similar, se buscó distribuirlos entre estos distintos niveles de ingreso evitando que se concentraran alrededor de solo una o dos categorías. Los grupos de ingreso se establecieron así: menos de $\$ 10,000$ mensuales, entre $\$ 10,000$ - $\$ 19,999$, entre $\$ 20,000-$ $\$ 39,999$, entre $\$ 40,000$ - $\$ 59,999$, entre $\$ 60,000$ - $\$ 79,999$, entre $\$ 80,000$ - $\$ 99,999$, y más de $\$ 100,000$. También, los respondientes fueron distribuidos en distintos niveles de estudios: secundaria, preparatoria, licenciatura y posgrado. En cada caso la persona entrevistada debía evaluar su banco principal (aquel que utilizaran con mayor frecuencia y/o en el que realizaran la mayor cantidad de operaciones bancarias).

El cuestionario se aplicó mediante entrevista persona a persona (un encuestador debía leer las preguntas y anotar las respuestas). Se debía verificar que el respondiente entendiera las instrucciones. Para ayudar al respondiente, el entrevistador podía enseñar las opciones de respuesta para que entendieran cómo deben elegir, pero en ningún momento se entregó el cuestionario al sujeto. Los entrevistadores no debían conocer a las personas o al menos no tener una relación directa o cercana con ellas. Los respondientes no debían ser parientes entre sí. Para motivar a las personas a responder la entrevista se les explicó que se trata de una investigación que se está llevando a cabo en el Tecnológico de Monterrey. Este trabajo de campo se llevó a cabo durante el mes de septiembre en 2012.

Después de una examinación cuidadosa de los cuestionarios llenados, hubo que eliminar a algunos debido a errores en la aplicación de las entrevistas. Así, se obtuvo información depurada de $n=201$ clientes de bancos detallistas. De esta manera, esta es la muestra que se utilizó para los análisis de datos que son presentados en las siguientes secciones. Para tener cierto control sobre la variabilidad potencialmente generada por la marca del banco, el estudio se cerró solo a clientes de cuatro marcas de bancos detallistas. Estas marcas fueron seleccionadas tomándolas con base en aquellas que tienden a tener las mayores participaciones de mercado (Asociación de Bancos de México, 2012). Así, la muestra está conformada por $41 \%$ de clientes cuyo banco principal reportaron como Banamex, 28\% de Bancomer, 20\% de HSBC y $11 \%$ de Santander.

\section{Pruebas de validez y confiabilidad}

Para examinar si los reactivos cuentan tanto con validez discriminante como con validez convergente, con los datos se llevó a cabo un análisis factorial con rotación varimax. Este tipo de rotación sirve para maximizar la separación de los reactivos en diferentes factores lo que resulta apropiado tanto para probar qué tanto algunos reactivos convergen en una misma dimensión (tenderían a medir lo mismo), y qué tanto se discriminan de reactivos que miden otra propiedad (Russell, 2002). En la Tabla 2 se muestra el resultado de este análisis. Se puede apreciar que se obtuvieron tres factores de forma natural (sin forzar el número de factores y con auto-valores mayores a 1) de forma nítida. Así, los reactivos convergieron en dimensiones que, al analizar el contenido de los reactivos, permiten una interpretación clara de las mismas. Todos los reactivos relacionados en la atención del personal del banco al cliente se juntaron en una dimensión que fue nombrada como "atención" (atención hacia el cliente). Los reactivos relacionados con el cumplimiento del servicio que el cliente espera recibir y por el cuál acude al banco convergieron en una dimensión que se le llamó "confiabilidad" (confiabilidad en la 
ejecución del servicio). Por último, los reactivos asociados a aspectos visuales como apariencia del equipo y de las instalaciones se tendieron a reunir en un componente que se denominó "aspecto/tangibles" (aspecto visual de los aspectos tangibles). Para valorar la validez estadística de este análisis factorial se realizó la prueba KMO y la prueba de esfericidad de Bartlett. La prueba KMO arrojó un valor alto de .96 y la prueba de esfericidad resultó estadísticamente significativa. Estos resultados tienden a mostrar que los factores resultantes explican a las variables observadas y por lo tanto que el modelo es aceptable (Tobias y Carlson, 1969; Dziuban y Shirkey, 1974).

Tabla 2

Análisis factorial para examinar convergencia y discriminación de los reactivos. Matriz de componentes rotados. Cargas factoriales.

\begin{tabular}{|c|c|c|c|}
\hline \multicolumn{4}{|c|}{ Componente } \\
\hline Reactivo & 1 Atención & 2 Confiabilidad & 3 Aspecto/Tangibles \\
\hline sqb20 & .761 & & \\
\hline sqb22 & .735 & & \\
\hline sqb14 & .714 & & \\
\hline sqb18 & .699 & & \\
\hline sqb17 & .693 & & \\
\hline sqb16 & .675 & & \\
\hline sqb12 & .674 & & \\
\hline sqb21 & .664 & & \\
\hline sqb19 & .630 & & \\
\hline sqb11 & .626 & & \\
\hline sqb13 & .621 & & \\
\hline sqb15 & .583 & & \\
\hline sqb6 & & .813 & \\
\hline sqb8 & & .800 & \\
\hline sqb9 & & .757 & \\
\hline sqb7 & & .717 & \\
\hline sqb5 & & .717 & \\
\hline sqb10 & & .688 & \\
\hline sqb2 & & & .879 \\
\hline sqb1 & & & .865 \\
\hline sqb3 & & & .636 \\
\hline sqb4 & & & .584 \\
\hline Alfa & .96 & .94 & .88 \\
\hline \multicolumn{4}{|c|}{$\begin{array}{l}\text { Método de extracción: componentes principales. Solución explica el } 72 \% \text { de la } \\
\text { variabilidad original. Método de rotación: Varimax con normalización Kaiser. } \\
\text { Rotación convergió en } 6 \text { iteraciones. Nivel de corte a .55. Alfa: coeficiente de } \\
\text { fiabilidad alfa de Cronbach. }\end{array}$} \\
\hline
\end{tabular}

Fuente: elaboración propia

En el análisis factorial que se muestra en la Tabla 2, sólo se muestran cargas factoriales (correlaciones de los reactivos con los factores resultantes) arriba de .55. De esta forma se puede ver una solución final muy nítida. Sin embargo, algunos de los reactivos llegan a mostrar cargas alrededor de 4 con algún otro factor (o dimensión). Esto puede hacer suponer que no existe completa independencia estadística entre las tres dimensiones. Esta idea es retomada más adelante en el análisis estructural confirmatorio. La forma en que los reactivos se asocian de forma diferenciada a cada factor permite argumenta la existencia tanto de validez convergente 
como de validez discrimínate en la medición de estas tres dimensiones de validad del servicio en bancos detallistas.

Adicionalmente, en la Tabla 2 se muestran los resultados del alfa de Cronbach para los reactivos dentro de cada una de las dimensiones obtenidas. Esto se presenta así debido a que existen evidencias que muestran que el alfa de Cronbach tiende a ser una prueba más adecuada para medir confiabilidad en un grupo de reactivos que miden una misma dimensión (Hair et al., 2010, p. 340). Los coeficientes alfa obtenidos tienden a indicar que se cuenta con una alta consistencia interna de los reactivos por cada dimensión que estaría midiendo esta escala. Valores superiores a 0.8 tienden a considerarse como muy buenos y superiores a .9 como excelentes (Gliem y Gliem, 2003).

Para medir la confiabilidad completa de toda la escala (los 22 reactivos) se obtuvo la Lamda de Guttman. La prueba de Guttman se ha argumentado como una adecuada (a diferencia del alfa de Cronbach) para determinar confiabilidad en una escala multidimensional con un gran número de reactivos (Hair et al., 2010, p. 340; Benton, 2015). Así, esta prueba realiza simultáneamente varias pruebas de mitades divididas donde separa los reactivos en dos grupos distintos de forma aleatoria y mide la consistencia entre ambos grupos (Benton, 2015). Los coeficientes obtenidos fueron entre .93 el más bajo y .98 el más alto. Los coeficientes de esta prueba se interpretan de forma similar que los del alfa de Cronbach (Hair et al., 2010, p. 341; Benton, 2015). Por lo que se puede concluir que esta prueba arroja indicadores altos de confiabilidad para esta escala.

Sin embargo, los resultados del análisis factorial mostrado en la Tabla 2 no coinciden exactamente con las dimensiones propuestas por Parasuraman et al. (1988) y luego retomadas por Cronin y Taylor (1992, 1994). Como se mencionó con anterioridad, ambos proponen cinco dimensiones (tangibles, fiabilidad, capacidad de respuesta, seguridad y empatía). Aparentemente, según el modelo obtenido de la Tabla 2, se estarían conjuntando en un solo componente (atención del personal) las dimensiones de: respuesta, seguridad y empatía. Se obtuvieron las dimensiones tangibles y confiabilidad lo que sí es consistente con estudios anteriores. De tal forma, se realizó otro análisis factorial, pero en esta ocasión se forzó a cinco componentes para si estos coinciden con las cinco dimensiones presentadas por Parasuraman et al. (1988) y por Cronin y Taylor $(1992,1994)$. En la Tabla 3 se ven los resultados obtenidos de este segundo análisis factorial. 
Tabla 3

Análisis factorial forzado a cinco componentes para examinar convergencia y discriminación de los reactivos. Matriz de componentes rotados. Cargas factoriales.

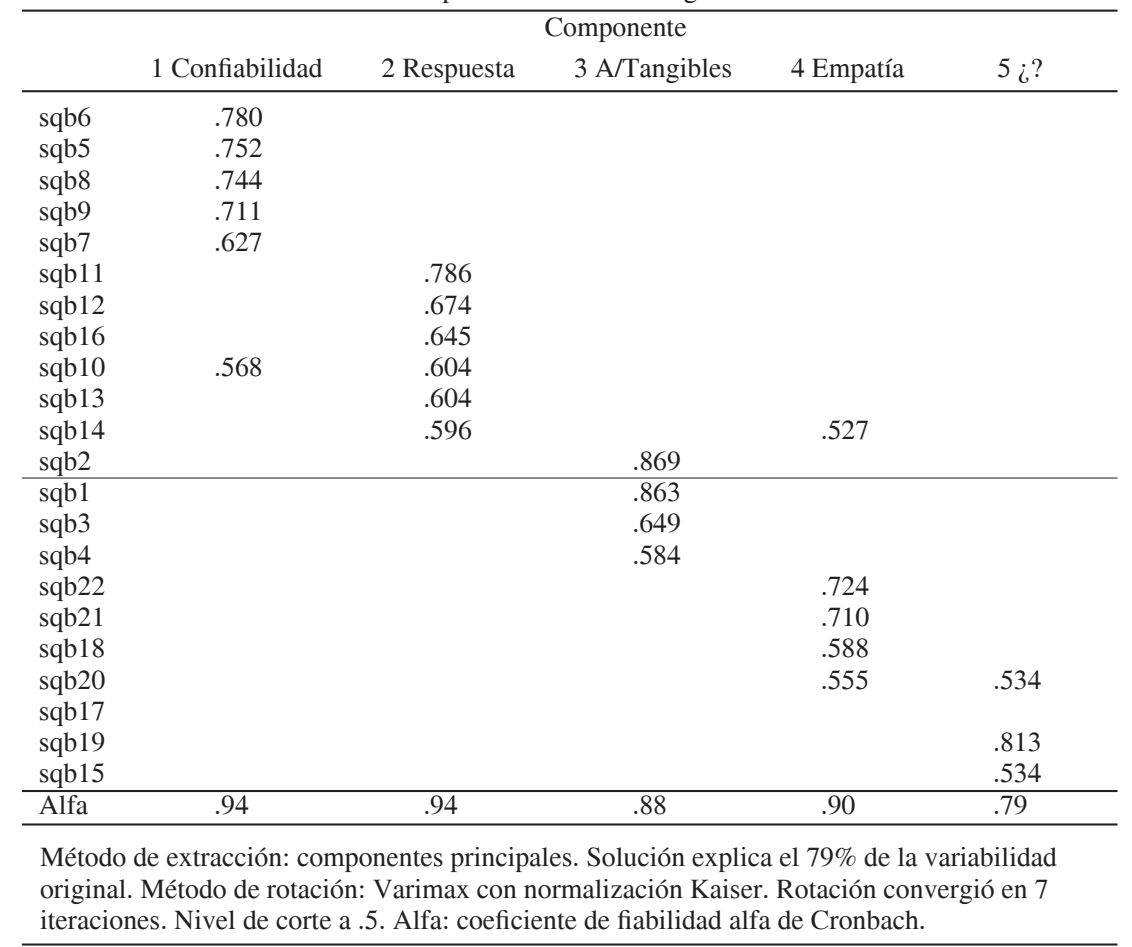

Fuente: elaboración propia

Aunque con este segundo análisis sí tiende a confirmar cuatro de las cinco dimensiones (tangibles, confiabilidad, capacidad de respuesta, y empatía), algunos reactivos se cargan a dimensión distintas a las esperadas (reactivos: 10, 14, 16, 20 y 19) y la dimensión seguridad es aislada con muy poca claridad con sólo dos de los cinco reactivos que debían converger (lo que sugiere que la solución de tres factores es más adecuada.).

\section{Modelo estructural confirmatorio}

Para reforzar las evidencias de validez discriminante y convergente de los reactivos con respecto a las tres dimensiones obtenidas se llevó a cabo un análisis confirmatorio de ecuaciones estructurales. El modelo obtenido se puede apreciar en la Figura 1. En este modelo todos los estimadores resultaron estadísticamente significativos al 0.001. En cuanto a los indicadores de ajuste del modelo se obtuvo un CMIN/DF de 2.68; de acuerdo a Wheaton et al. (1977) valores inferiores a 5 se pueden considerar aceptables. En el indicador RMSEA se obtuvo un valor de .092, aquí, de acuerdo a Browne y Cudeck (1993) aunque preferentemente debe tener un valor inferior a 0.08 para mostrar un ajuste adecuado de los datos al modelo, pueden aceptarse valores inferiores a 0.1 . Con respecto a los índices de bondad de ajuste IFI y CFI el modelo alcanzó valores de .92 y .91 respectivamente, aunque en los otros índices de este tipo NFI, y TLI se obtuvieron valores ligeramente por debajo de 0.9 (.87 y .89); de acuerdo a Bentler y 
Bonett (1980) se deben alcanzar valores de 0.9 o superiores para considerar que se tiene un ajuste del modelo a la realidad adecuado. Aunque en general los datos de estimación y ajuste del modelo obtenido no son los mejores, permiten argumentar con reservas que el modelo estructural puede ser aceptable.

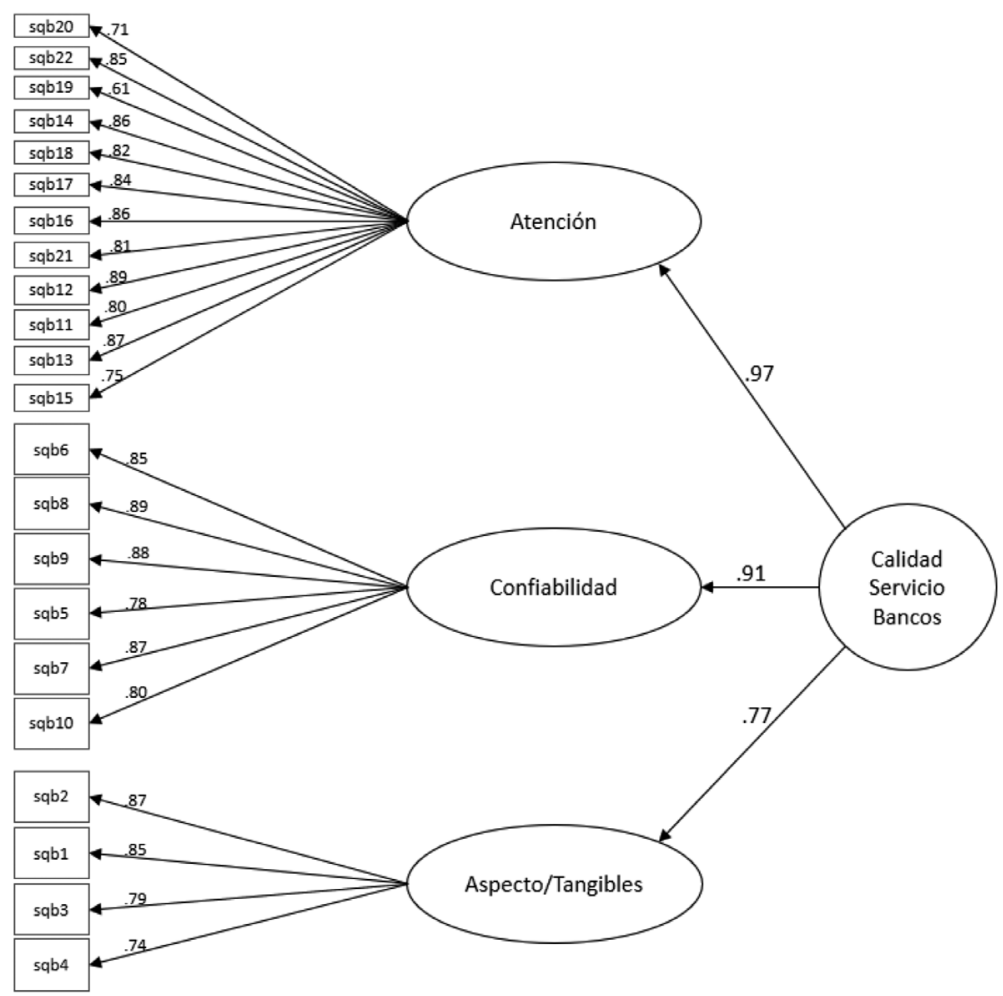

Figura 1. Modelo estructural. Pesos estructurales y pesos de medición. Modelo de veintidós variables observadas, tres variables latentes de primer orden y una variable latente de segundo orden.

El modelo estructural obtenido tiende a confirmar la existencia de las tres dimensiones obtenidas en el análisis factorial (empatía, seguridad y aspecto). Adicionalmente, se tiende a confirmar la existencia de una variable latente de segundo orden en donde convergen las tres anteriores. En este modelo (Figura 1) se puede observar que en los estimadores de medición (correlaciones entre las variables observadas y las variables latentes de primer orden) se obtienen en general valores altos superiores a 0.7 y en muchos casos superiores a 0.8 lo que tendería a confirmar la validez convergente de los reactivos en sus respectivas dimensiones. De esta manera se tiene que la variable latente de segundo orden (calidad en el servicio) estima de forma adecuada a cada una de las variables latentes de primer orden (empatía, seguridad y aspecto) y estás últimas a su vez estiman de forma adecuada a las variables observadas (reactivos de calidad en el servicio en bancos). Con esto se tienen evidencias que los reactivos 
tienden a converger de forma adecuada en alguna de las tres dimensiones y a discriminarse de las otras dos, y a su vez que las tres dimensiones convergen en una dimensión superior, indicando que se tiene un constructo multifactorial de calidad en el servicio en bancos.

Se planteó un segundo modelo estructural. Uno que fuera consistente con las cinco dimensiones presentadas por Parasuraman et al. (1988) y por Cronin y Taylos (1992 y 1994) tal como ellos agruparon los reactivos. El diagrama de este modelo junto con sus estimadores puede verse en la Figura 2. No se tenían buenas expectativas para este modelo dado lo obtenido en el segundo análisis factorial donde se intentó confirmar estas cinco dimensiones (ver Tabla 3). No obstante, los datos se ajustaron al modelo de forma admisible. Los indicadores de ajuste CMIN/DF y RMSEA dieron valores aceptables de 2.35 y .082 respectivamente. Todos los indicadores de bondad de ajuste, menos uno, también dieron valores aceptables $(\mathrm{NFI}=.89$, $\mathrm{RFI}=.86, \mathrm{IFI}=.93, \mathrm{TLI}=.92$ y $\mathrm{CFI}=.93$ ). Este modelo de cinco dimensiones (variables latentes de primer orden) tiende a mostrar pesos estructurales y pesos de medición altos (significativos) implicando nuevamente validez por convergencia y por discriminación de los reactivos.

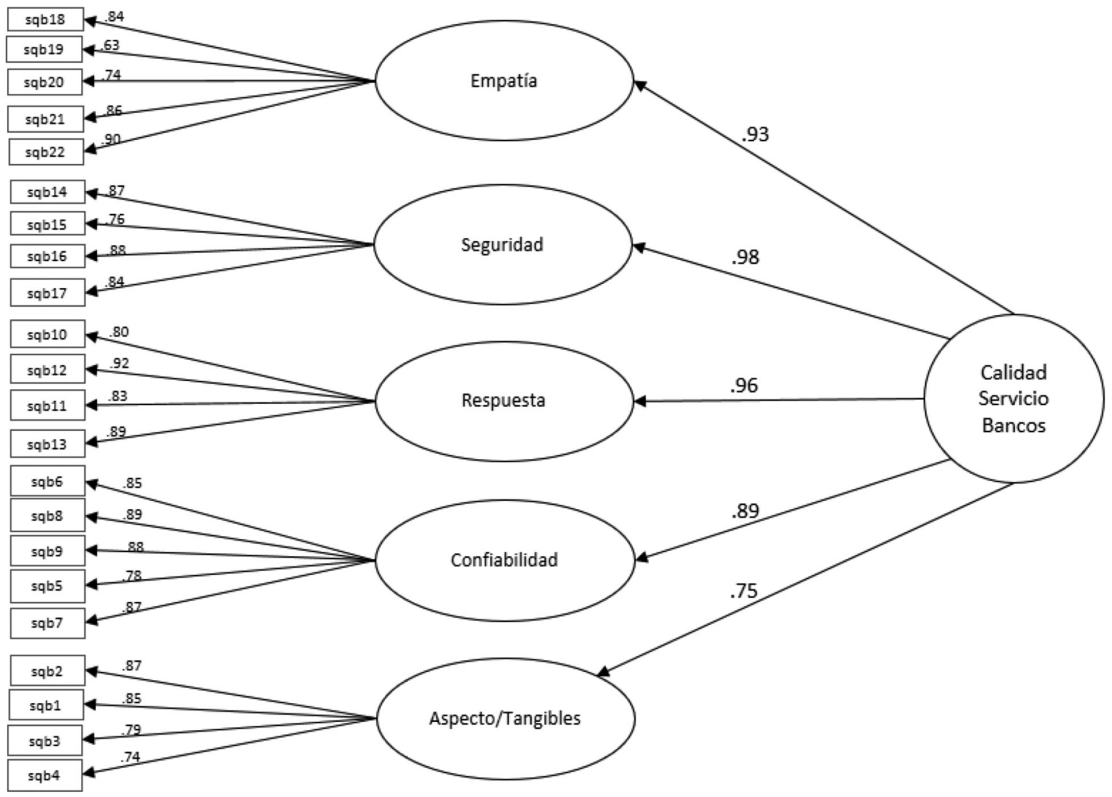

Figura 2. Modelo estructural. Pesos estructurales y pesos de medición. Modelo de veintidós variables observadas, cinco variables latentes de primer orden y una variable latente de segundo orden.

\section{Validez predictiva}

Con el propósito de ofrecer evidencias de la capacidad de validez predictiva del instrumento, en el cuestionario que se utilizó para este levantamiento de datos se incluyó un reactivo indicador del grado general de satisfacción del cliente hacia el banco. Este reactivo dice: "En general me siento satisfecho con los servicios que me proporciona el banco". En el cuestionario 
este reactivo fue asociado a una escala de actitud de siete posiciones que van de totalmente en desacuerdo a totalmente de acuerdo. Reactivos similares a este, para medir satisfacción, han sido ampliamente utilizados como en los casos de Zeithaml et al. (1996), Jamal y Naser (2002), Bauman et al. (2007), y Ryu et al. (2012).

Tabla 4

Análisis de regresión. Variable dependiente (Y): En general me siento satisfecho con los servicios que me proporciona el banco.

\begin{tabular}{ll}
\hline R (coeficiente de regresión múltiple) & .75 \\
R2 (coeficiente de determinación) & .55 \\
F (de Fisher para validez el modelo) & $80.9 * *$ \\
X1 Atención (coeficiente beta estandarizado) & $.42 * *$ \\
X2 Confiabilidad (coeficiente beta estandarizado) & $.58^{* *}$ \\
X3 Aspecto/Tangibles (coeficiente beta estandarizado) & $.21 * *$
\end{tabular}

**Significancia al 0.01 en la prueba t para los coeficientes de las variables independientes

Fuente: elaboración propia

Para presentar la prueba de validez predictiva se llevó a cabo un análisis de regresión lineal. En este análisis se utilizó como variable dependiente (variable a predecir) el reactivo de satisfacción general mencionado en el párrafo anterior. Como variables independientes se utilizaron los puntajes factoriales de los tres componentes de calidad en el servicio obtenidos del análisis factorial mostrado en la Tabla 2. Si la escala posee validez de predicción entonces los tres componentes de calidad en el servicio deben ser capaces de explicar la satisfacción general del cliente. La calidad del servicio como un constructo antecedente de la satisfacción del cliente en el sector bancario ha sido ampliamente confirmado con anterioridad como puede verse en: Levesque y McDougall (1996), Holmlund y Kock (1996), Jamal y Naser (2002), Lewis y Soureli (2006), Kumar et al. (2010), Manimaran (2010), Culiberg y Rojsek (2010), Ehsan et al. (2011).

En la Tabla 4 se muestran los resultados del análisis de regresión. Aquí se puede confirmar como los tres componentes de calidad del servicio de bancos detallistas tienen un efecto significativo positivo sobre la satisfacción general del cliente explicando un total del 55\% de la variabilidad. De forma particular se encontró que el factor (dimensión) confiabilidad del servicio, por su coeficiente estandarizado, es la variable independiente con mayor efecto seguido por atención del personal.

\section{Conclusiones}

Como puede verse en la sección referente a los análisis estructurales, se puede argumentar tanto la existencia de un modelo de tres dimensiones como uno de cinco en esta adaptación del instrumento SERVPERF. Si bien, en el modelo mostrado en la Figura 2, se muestra que los reactivos convergen en cinco dimensiones como lo propone originalmente Parasuraman et al. (1988) (empatía, seguridad, capacidad de respuesta, confiabilidad y elementos tangibles), el modelo representado en la Figura 1 muestra a su vez que empatía, seguridad y respuesta, al ser aspectos relacionados con lo que hace el personal del banco para los clientes, tienden a tener cierta relación más estrecha entre sí que con las otras dos dimensiones (confiabilidad y tangibles/aspecto). Es razonable pensar que la empatía que siente un cliente por parte del personal del banco puede estar fuertemente influenciada por la pronta respuesta y la claridad 
que el personal muestre hacia él. Por el otro lado, los reactivos que componen las dimensiones confiabilidad y tangibles/aspecto hacen referencia a aspecto del banco como organización más que al personal que ahí labora. En este sector de bancos detallistas, la solución del modelo de tres dimensiones de desempeño del servicio como aquí se han presentado es también confirmada por estudios anteriores donde se han obtenido resultados similares (Karatepe et al., 2005; Culiberg y Rojsek, 2010). Así, se ha encontrado anteriormente que las dimensiones de las escalas basadas en SERVQUAL pueden variar por tipo de servicio, así como por país, y no necesariamente se van a confirmar las cinco originales (Babakus y Boller, 1992; Jabnoun y Khalifa, 2005: Akbaba, 2006).

El SERVPERF al ser un instrumento generalista pensado para ser aplicado a una gran cantidad de industrias contempla variables que en términos generales tienden a ser relevantes para muchos sectores. Sin embargo, previamente se ha discutido de forma amplia las limitaciones de este tipo de enfoque. Estas limitaciones se refieren esencialmente a que no se toman aspectos muy particulares que pueden ser muy importantes sólo para algún sector por lo que muchas veces se requiere adicionar con nuevas variables a las escalas fundadas en el SERVQUAL y en el SERVPERF (Van Dyke, et al., 1997; Jabnoun y Khalifa, 2005). De tal forma, si se va a utilizar la presente escala para medir desempeño del servicio en bancos detallistas se recomienda, además de los veintidós reactivos aquí presentados, adicionar la escala con reactivos de aspecto muy específicos de este tipo de negocios. De esta manera, Culiberg y Rojsek (2010) además de utilizar los reactivos de desempeño del SERVQUAL, detectan una sexta dimensión del servicio de bancos que le llaman "accesibilidad" donde elaboran reactivos para medir desempeño de tres nuevos atributos: conveniencia del horario de servicio, facilidad de estacionamiento, localización conveniente de la sucursal, y amplitud de los servicios (productos) que ofrece el banco. Esto lo hacen con los reactivos ${ }^{1}$ :

1. "El horario en el que opera el banco se acomoda a mis necesidades"

2. "El acceso al banco es fácil (estacionamiento)"

3. "La ubicación del banco me es favorable"

4. "La diversidad de productos financieros que ofrece el banco se ajusta a mis necesidades"

En este mismo orden de ideas, Merino (2001) proponen medir, adicionalmente a los elementos ya contemplados en las dimensiones del SERVPERF/SERVQUAL, factores específicos del servicio bancario como: Imagen de los productos y servicios; Funcionamiento de cajeros y equipos; y flexibilidad. De tal forma, se podrían elaborar reactivos de los aspectos sugeridos por este autor como son:

1. "Buena reputación de la entidad"

2. "Credibilidad"

3. "Solidez y fiabilidad financiera"

4. "Condiciones financieras ventajosas: tipos de interés, plazos amortización, comisiones"

5. "Adaptación de los productos y servicios financieros ofrecidos a las necesidades del cliente”

6. “Amplia gama de productos y servicios para cubrir las necesidades del cliente”

\footnotetext{
${ }^{1}$ Estas son las traducciones simples de los reactivos que proponen los autores Culiberg y Rojsek (2010), no han sido adaptados ni probados estadísticamente para validez y confianza en el contexto mexicano.
} 
7. "Correcto funcionamiento de los equipos técnicos instalados"

8. "Correcto funcionamiento y disponibilidad de fondos en cajeros automáticos"

9. "Horario de atención al público suficientemente flexible"

10. "La entidad evita, en lo posible, excesivas garantías, avales y papeleo"

De esta manera, si en un estudio se van a utilizar los veintidós reactivos aquí presentados, se sugiere incluir estos catorce reactivos adicionales para contemplar estos atributos específicos. Así, para estudios a futuro, se sugiere hacer una detección de atributos deseables en el contexto mexicano no contemplado actualmente y desarrollar los respectivos reactivos (validez y confiabilidad) para enriquecer la presente escala.

Los reactivos aquí presentados, si bien pueden ser usados para estudios académicos, también pueden ser utilizados para realizar estudios gerenciales en el caso, por ejemplo, donde un banco en México quiera medir la calidad en el servicio que se está prestando en sus diferentes sucursales. Asimismo, también puede ser utilizado en el caso de que se quiera hacer comparativos del desempeño del servicio entre distintas marcas de bancos con el fin de detectar la forma en que distintos competidores son percibidos por sus clientes en cuanto al servicio que prestan. Así, este instrumento puede ser utilizado en una amplia diversidad de estudios.

Como ya se ha establecido, la presente escala sólo sirve para medir calidad del servicio en la atención directa a clientes en una sucursal bancaria. Dada la importancia que ha tomado el servicio de banco detallista por internet, surge la oportunidad para un estudio a futuro donde se desarrolle una escala para medir calidad del servicio bancario en este medio en México. Esta escala deberá conformarse por un grupo de reactivos y dimensiones diferentes a la escala que aquí se ha presentado. Estos atributos deberán tener relación con los componentes de atención al cliente mediante los portales de banca por internet de los diferentes bancos. De esta manera, como antecedentes para un nuevo estudio, y como fuentes posibles de reactivos para la medición de calidad del servicio de banca por internet, se puede mencionar a: Han y Baek (2004); a Rod et al. (2009); y a Ho y Lin (2010).

\section{Agradecimientos}

Se agradece al Dr. Frank Pons de la Universidad Laval en Quebec (Canadá) por habernos facilitado la versión en inglés de la adaptación del SERVPERF a bancos detallistas de la cual se partió para llevar a cabo el presente trabajo. También se desea agradecer al equipo de becarios de la carrera de mercadotecnia en la Escuela de Negocios del Campus Ciudad de México del Tecnológico de Monterrey por su valiosa colaboración en el levantamiento de datos que da soporte a este estudio. 


\section{Referencias}

Akbaba, A. (2006). Measuring Service Quality in the Hotel Industry: A Study in a Business Hotel in Turkey. International Journal of Hospitality Management, 25 (2): 170-192. https://doi.org/10.1016/j.ijhm.2005.08.006

Andaleeb, S. y Conway, C. (2006). Customer satisfaction in the restaurant industry: an examination of the transaction-specific model. The Journal of Services Marketing, 20 (1): 3-11. https://doi.org/10.1108/08876040610646536

Antun, J., Fras, R., Costen, W. y Runryan, R. (2010). Accurately Assessing Expectations Most Important to Restaurant Patrons: The Creation of the DinEX Scale. Journal of Foodservice Business Research, 13 (4) https://doi.org/10.1 080/15378020.2010.524539

Asociación de Bancos de México (2012). Financial groups balance. Disponible en: http://www.abm.org.mx/banca_ mexico/estadisticas.htm). Consultado: 24/07/2016.

Babakus, E. y Boller, G.W. (1992). An empirical assessment of the SERVQUAL scale. Journal of Business Research, 24 (3): 253-268. https://doi.org/10.1016/0148-2963(92)90022-4

Baumann, Chris, Suzan Burton, Gregory Elliott and Hugo M. Kehr, (2007). Prediction of attitude and behavioural intentions in retail banking. International Journal of Bank Marketing, 25 (2): 102-116. https://doi. org/10.1108/02652320710728438

Bentler, P.M. y Bonett, D.G. (1980). Significance tests and goodness of fit in the analysis of covariance structures. Psychological Bulletin, 88 (3): 588-606. https://doi.org/10.1037//0033-2909.88.3.588

Browne, M.W. y Cudeck, R. (1993), Alternative ways of assessing model fit. In Bollen, K.A. y Long, J.S., Testing structural equation models, Newbury Park, CA: Sage, 136-162.

Belás, J., y Gabcová, L. (2016). The relationship among customer satisfaction, loyalty and financial performance of commercial banks. E+M Ekonomie a Management, (1): 132-147. https://doi.org/10.15240/tul/001/2016-1-010

Benton, T. (2015). An empirical assessment of Guttman's Lambda 4 reliability coefficient. Quantitative Psychology Research (pp. 301-310): Springer International Publishing. https://doi.org/10.1007/978-3-319-07503-7_19

Brady, M. K., Cronin, J.J., y Brand, R.R. (2002). Performance only measurement of service quality: a replication and extension. Journal of Business Research, 55 (1): 17-31. https://doi.org/10.1016/s0148-2963(00)00171-5

Cater-steel, A., y Lepmets, M. (2014). Measuring IT service quality: Evaluation of IT service quality measurement framework in industry. Journal of Service Science Research, 6 (1): 125-147. https://doi.org/10.1007/s12927-0140005-5

Carrillat, F. A., Jaramillo, F. y Mulki, J. P. (2007). The validity of the SERVQUAL and SERVPERF scales. International Journal of Service IndustryManagement, 18(5):472-490.https://doi.org/10.1108/09564230710826250

Comisión Nacional Bancaria y de Valores, (2016). Información operativa, Banca Múltiple, 040-4ª-R1, Información de sucursales, cajeros automáticos y tarjetas de crédito. Consultado en: http://portafoliodeinformacion.cnbv.gob.mx/ bm1/Paginas/infoper.aspx

Cronin, J. J. y Taylor, S. A. (1992). Measuring service quality: a reexamination and extension. Journal of Marketing, 56(3): 55-68. https://doi.org/10.2307/1252296

Cronin, J. J. y Taylor, S. A. (1994). SERVPERF versus SERVQUAL; reconciling performance-based. Journal of Marketing, 58 (1): 125-131. https://doi.org/10.2307/1252256

Culiberg, B., y Rojšek, I. (2010). Identifying service quality dimensions as antecedents to customer satisfaction in retail banking. Economic and Business Review, 12 (3): 151-166.

Dziuban, C. D., y Shirkey, E. C. (1974). When is a correlation matrix appropriate for factor analysis? Some decision rules. Psychological Bulletin, 81 (6): 358-361. https://doi.org/10.1037/h0036316

Ehsan, M., Naeem, B., and Arif, Z. (December 2011). How do service quality perceptions contribute in satisfying banking customers?. Interdisciplinary Journal of Contemporary Research in Bussiness, 3 (8): 646-653.

Gliem, J. A., y Gliem, R. R. (2003). Calculating, interpreting, and reporting Cronbach's alpha reliability coefficient for Likert-type scales. Midwest Research-to-Practice Conference in Adult, Continuing, and Community Education.

Grönroos, Ch. (1984). A service quality model and its marketing implications. European Journal of Marketing, 18 (4): 36-44. https://doi.org/10.1108/eum0000000004784 
Hair, J. F., Bush, R. P. y Ortinau, D. J. (2010). Investigación de mercados: en un ambiente de información digital, (4a Ed.), México, D.F.: McGraw-Hill, 651 pp.

Han, S. L., y Baek, S. (2004). Antecedents and consequences of service quality in online banking: An application of the SERVQUAL instrument. NA-Advances in Consumer Research, 31 (1): 208-214.

Ho, C. T. B., \& Lin, W. C. (2010). Measuring the service quality of internet banking: scale development and validation. European Business Review, 22 (1): 5-24. https://doi.org/10.1108/09555341011008981

Holmlund, M. y Kock, S. (1996). Relationship Marketing: the Importance of Customer-Perceived Service Quality in Retail Banking. The Service Industries Journal, 16 (3): 287-304. https://doi.org/10.1080/02642069600000029

INEGI (2012). Encuesta Nacional de Inclusión Financiera. Disponible en: http://www.cnbv.gob.mx/Inclusi\%C3\%B3n/ Documents/Folleto\%20Resultados\%20ENIF2012.pdf. Consultado en: 20/03/2016.

Jabnoun, N. y Khalifa, A. (2005). A customized measure of service quality in the UAE. Managing Service Quality, 15(4): 374-388. https://doi.org/10.1108/09604520510606844

Jain, R., Sahney, S., y Sinha, G. (2013). Developing a scale to measure students' perception of service quality in the indian context. TQM Journal, 25 (3): 276-294. https://doi.org/10.1108/17542731311307456

Jain, S.K. y Gupta, G. (2004). Measuring service quality: SERVQUAL vs. SERVPERF scales. Vikalpa: The Journal for Decision Makers, 29(2), 25-37. https://doi.org/10.1177/0256090920040203

Jamal, A. y Naser, K. (2002). Customer satisfaction and retail banking: an assessment of some of the key antecedents of customer satisfaction in retail banking. International Journal of Bank Marketing, 20 (4): 146- 160. https://doi. org/10.1108/02652320210432936

Karatepe, O. M., Yavas, U., \& Babakus, E. (2005). Measuring service quality of banks: Scale development and validation. Journal of Retailing and Consumer Services, 12 (5): 373-383. https://doi.org/10.1016/j.jretconser.2005.01.001

Kaur, N., y Kiran, R. (2015). E-banking service quality and customer loyalty: Changing dynamics of public, private and foreign bank consumers in India. Global Business and Management Research, 7 (1): 74-92.

Keith, N. K., y Simmers, C. S. (2013). Measuring hotel service quality perceptions: the disparity between comment cards and lodgserv. Academy of Marketing Studies Journal, 17 (2): 119-131.

Kumar, S. A., Mani, B. T., Mahalingam, S., y Vanjikovan, M. (2010). Influence of Service Quality on Attitudinal Loyalty in Private Retail Banking: An Empirical Study. The IUP Journal of Management Research, 9 (4): 21-38.

Ladhari, R. (2008). Alternative measures of service quality: a review. Managing Service Quality, 18(1), 65-86. https:// doi.org/10.1108/09604520810842849

Ladhari, R., Pons, F., Bressolles, G., \& Zins, M. (2011). Culture and personal values: How they influence perceived service quality. Journal of Business Research, 64 (9): 951-957. https://doi.org/10.1016/j.jbusres.2010.11.017

Levesque, T. y McDougall, H. G. (1996). Determinants of customer satisfaction in retail banking. International Journal of Bank Marketing, 14 (7): 12-20. https://doi.org/10.1108/02652329610151340

Lewis, B. R., and Soureli, M. (2006). The antecedents of consumer loyalty in retail banking. Journal of Consumer Behaviour, 5 (1): 15-31. https://doi.org/10.1002/cb.46

Manimaran, S. (May-August 2010). Linkage between service quality and customers loyalty in commercial banks. Journal of Marketing \& Comunication, 6 (1): 26-34.

Marino, S. (2001). La calidad de servicio bancario: una escala específica de medida. Esic Market, Septiembre - Diciembre: $119-141$

Mittal, S., Gera, R., y Batra, D. K. (2015). An evaluation of an integrated perspective of perceived service quality for retail banking services in India. The International Journal of Bank Marketing, 33 (3): 330-350. https://doi. org/10.1108/ijbm-02-2014-0020

Padma, P., Sai L.P. y Rajendran C. (2015). Customer Satisfaction in Indian Hospitals: Moderators and Mediators. The Quality Management Journal, 22 (1): 10-29. https://doi.org/10.1080/10686967.2015.11918416

Parasuraman, A., Zeithaml, V. y Berry, L. L. (1985). A conceptual model of service quality and its implications for future research. Journal of marketing, 49 (4): 41-50. https://doi.org/10.2307/1251430

Parasuraman, A., Zeithaml, V. A., y Berry, L. L. (1988). SERVQUAL. Journal of retailing, 64 (1): 12-40.

Raajpoot, N. (2004). Reconceptualizing service encounter quality in a non-western context. Journal of Service Research, 7 (2): 181-201. https://doi.org/10.1177/1094670504268450 
Radomir, L., Plaias, I. y Nistor, V. (2012). A review of the service quality concept-past, present and perspectives. Marketing-from information to decision, 5 (1): 404-427.

Rauch, D. A., Collins, M. D., Nale, R. D., y Barr, P. B. (2015). Measuring service quality in mid-scale hotels. International Journal of Contemporary Hospitality Management, 27 (1): 87-106. https://doi.org/10.1108/ijchm-06-2013-0254

Rod, M., Ashill, N. J., Shao, J., \& Carruthers, J. (2009). An examination of the relationship between service quality dimensions, overall internet banking service quality and customer satisfaction: A New Zealand study. Marketing Intelligence \& Planning, 27 (1): 103-126. https://doi.org/10.1108/02634500910928344

Russell, D. W. (2002). In search of underlying dimensions: The use (and abuse) of factor analysis in Personality and Social Psychology Bulletin. Personality and social psychology bulletin, 28 (12): 1629-1646. https://doi. org/10.1177/014616702237645

Ryu, K., Lee H. R. and Gon Kim, W. (2012). The influence of the quality of the physical environment, food, and service on restaurant image, customer perceived value, customer satisfaction, and behavioral intentions. International Journal of Contemporary Hospitality Management, 24 (2): 200-223. https://doi.org/10.1108/09596111211206141

Simmers, C. S., y Keith, N. K. (2015). Measuring retail store service quality: the disparity between the retail service quality scale (RSQS) and comment cards. Academy of Marketing Studies Journal, 19 (2): 117-125.

Tobias, S., y Carlson, J. E. (1969). Brief report: Bartlett's test of sphericity and chance findings in factor analysis. Multivariate Behavioral Research, 4 (3): 375-377. https://doi.org/10.1207/s15327906mbr0403_8

Van Dyke, T. P., Kappelman, L. A. y Prybutok, V. R. (1997). Measuring information systems service quality: concerns on the use of the SERVQUAL questionnaire. MIS Quarterly, 21(2): 195-208. https://doi.org/10.2307/249419

Wheaton, B., Muthén, B., Alwin, D. F. y Summers, G. F. (1977). Assessing reliability and stability in panel models. In Heise, D. R. (1977). Sociológical methodology, San Francisco: Jossey-Bas: 84-136. https://doi.org/10.2307/270754

Zeithaml, V. A., Leonard L. B. and A. Parasuraman (April 1996). The Behavioral Consequences of Service Quality. Journal of Marketing, 60 (2): 31-46. https://doi.org/10.2307/1251929

Zeithaml, V. A. (2000). Service quality, profitability, and the economic worth of customers: What we know and what we need to learn. Academy of Marketing Science Journal, 28 (1): 67-85. https://doi.org/10.1177/0092070300281007 


\section{Apéndice 1 \\ Cuestionario}

En este cuestionario XYZ representa su principal banco proveedor de servicios bancarios (por favor anotar nombre del banco: J.

\section{Parte 1: calidad en el servicio}

Las declaraciones que se presentan a continuación se relacionan con la posible opinión que usted pueda tener del servicio que le provee el banco XYZ. Para cada declaración, por favor indique en qué grado usted considera que el banco XYZ cumple con la característica descrita en cada declaración. Los números 1 al 7 representan un continuo donde 1 significa que estaría totalmente en desacuerdo y 7 que estaría totalmente de acuerdo. En cada caso circule el número que mejor refleje su opinión acerca del cumplimiento del banco acerca de cada declaración. No existen respuestas correctas o incorrectas, lo único que nos interesa es contar con algunos datos sobre la percepción general hacia la calidad en el servicio ofrecida por su banco principal.

\begin{tabular}{|c|c|c|c|c|c|c|c|}
\hline \multirow[b]{2}{*}{ 1- El banco $X Y Z$ tiene un equipamiento que se ve moderno. } & \multicolumn{5}{|c|}{$\begin{array}{l}\text { Totalmente } \\
\text { en desacuerdo }\end{array}$} & \multicolumn{2}{|c|}{$\begin{array}{l}\text { Totalmente } \\
\text { de acuerdo }\end{array}$} \\
\hline & 1 & 2 & 3 & 4 & 5 & 6 & 7 \\
\hline 2- Las instalaciones del banco XYZ son visualmente atractivas. & 1 & 2 & 3 & 4 & 5 & 6 & 7 \\
\hline $\begin{array}{l}\text { 3- Los empleados del banco XYZ presentan una apariencia muy bien } \\
\text { cuidada. }\end{array}$ & 1 & 2 & 3 & 4 & 5 & 6 & 7 \\
\hline $\begin{array}{l}\text { 4- La folletería y otros materiales impresos del banco XYZ son visualmente } \\
\text { atractivos. }\end{array}$ & 1 & 2 & 3 & 4 & 5 & 6 & 7 \\
\hline 5- Cuando el banco $X Y Z$ promete hacer algo en cierto tiempo, lo cumple. & 1 & 2 & 3 & 4 & 5 & 6 & 7 \\
\hline $\begin{array}{l}\text { 6- Cuando usted tiene un problema, el banco XYZ muestra un interés } \\
\text { sincero en resolverlo. }\end{array}$ & 1 & 2 & 3 & 4 & 5 & 6 & 7 \\
\hline 7-El banco XYZ realiza bien el servicio a la primera vez & 1 & 2 & 3 & 4 & 5 & 6 & 7 \\
\hline 8- El banco XYZ cumple con sus servicios en el tiempo prometido. & 1 & 2 & 3 & 4 & 5 & 6 & 7 \\
\hline 9- El banco $X Y Z$ busca siempre en no cometer errores. & 1 & 2 & 3 & 4 & 5 & 6 & 7 \\
\hline $\begin{array}{l}\text { 10- Los empleados del banco XYZ le dicen exactamente en qué tiempo se } \\
\text { cumplirá el servicio. }\end{array}$ & 1 & 2 & 3 & 4 & 5 & 6 & 7 \\
\hline 11- Los empleados del banco XYZ le brindan un servicio rápido. & 1 & 2 & 3 & 4 & 5 & 6 & 7 \\
\hline 12- Los empleados del banco XYZ siempre están dispuestos a ayudarlo. & 1 & 2 & 3 & 4 & 5 & 6 & 7 \\
\hline $\begin{array}{l}\text { 13- Los empleados del banco XYZ siempre tienen tiempo de responder a } \\
\text { sus solicitudes. }\end{array}$ & 1 & 2 & 3 & 4 & 5 & 6 & 7 \\
\hline $\begin{array}{l}\text { 14- E comportamiento de los empleados del banco XYZ genera confianza } \\
\text { en los clientes. }\end{array}$ & 1 & 2 & 3 & 4 & 5 & 6 & 7 \\
\hline 15- Se siente seguro al realizar transacciones en el banco $X Y Z$. & 1 & 2 & 3 & 4 & 5 & 6 & 7 \\
\hline 16- Los empleados del banco $\mathrm{XYZ}$ son siempre corteses con usted. & 1 & 2 & 3 & 4 & 5 & 6 & 7 \\
\hline $\begin{array}{l}\text { 17- Los empleados del banco XYZ tienen el conocimiento necesario para } \\
\text { responder a sus preguntas. }\end{array}$ & 1 & 2 & 3 & 4 & 5 & 6 & 7 \\
\hline 18- E banco $\mathrm{XYZ}$ le brinda atención personalizada. & 1 & 2 & 3 & 4 & 5 & 6 & 7 \\
\hline $\begin{array}{l}\text { 19- E banco XYZ tiene horarios de atención convenientes para sus. } \\
\text { clientes. }\end{array}$ & 1 & 2 & 3 & 4 & 5 & 6 & 7 \\
\hline 20- $\mathrm{E}$ banco $\mathrm{XYZ}$ tiene empleados que lo atienden personalmente. & 1 & 2 & 3 & 4 & 5 & 6 & 7 \\
\hline 21- Al banco XYZ realmente le importan sus necesidades. & 1 & 2 & 3 & 4 & 5 & 6 & 7 \\
\hline 22- Los empleados del banco XYZ entienden sus necesidades específica & 1 & 2 & 3 & 4 & 5 & 6 & 7 \\
\hline
\end{tabular}

